\title{
USO DE UN PROTOCOLO ESTANDARIZADO EN 14 PAÍSES PARA IDENTIFICAR FACTORES QUE AFECTAN LA EFICIENCIA DE LOS SERVICIOS DE INSEMINACIÓN ARTIFICIAL EN GANADO BOVINO A TRAVÉS DE ANÁLISIS DE PROGESTERONA ${ }^{1}$
}

\author{
M ario García P. ${ }^{2}$, W.J . Goodger, T. Bennett ${ }^{3}$ y B.M .A.O. Perera ${ }^{4}$
}

RESUMEN

El propósito del estudio fue cuantificar los principales factores limitantes que afectan los servicios de inseminación artificial (IA) en ganado bovino criado en países en desarrollo, a fin de recomendar estrategias para la mejora del porcentaje de concepción y el número de usuarios de la técnica delA. Seutilizó una metodol ogía de trabajo estandarizada en 14 países por un periodo de 5 años (1995-1999). Los países participantes fueron: Bangladesh, China, Indonesia, Myanmar, Pakistán, Sri Lanka y Vietnamen Asia; y Argentina, Chile, Costa Rica, Cuba, Penú, Uruguay y Venezuela en América Latina. Se programó hacer un seguimiento a un mínimo de 500 vacas de primer servicio postparto en cada país participante. Se recolectó información concerniente a las fincas, inseminadores, semen utilizado, vacas inseminadas, características de la expresión de celo y aspectos relacionados a la IA. Se tomaron 3 muestras de leche (o sangre en caso de vaquillas y ganado de carne) por cada inseminación realizada para medir progesterona por e método de radioinmunoensayo. Las muestras fueron col ectadas en el día de servicio (día 0) y a los 10-12 y 22-24 días posteriores al servicio. La información de campo y de laboratorio se registró en el programa de cómputo AIDA (Artificial Insemination DatabaseApplication). El estudio permitió determinar el estado delos servicios delA en los páses partici pantes y pudo mostrar importantes diferencias entre sistemas de producción de Asia y América Latina. El promedio \pm d.e del interval o entre el parto y el primer servicio para 7,992 observaciones fue de 120.0 \pm 82.1 días (mediana $=95$ días) con grandes diferencias entre países $(P \varangle 0.05)$. El porcentaje gl obal de concepción al primer servicio fue de $40.9 \%$ ( $n=8,196)$, siendo los Servicios de IA más eficientes el de Vietnam $(62.1 \%)$, Chile (61.9\%) y Myanmar (58.9\%). El interval o entre el primer y el segundo servicio fue $44.6 \pm 44.4$ días $(n=1,959)$. La combinación de niveles de progesterona en leche con información clínica pudo demostrar queel $17.3 \%$ de los servicios se real izó en vacas que no estaban ciclando (el $10.4 \%$ estaba en anestro, y el $4.7 \%$ estaba gestando). La mayor incidencia de problemas se observó en predi os ganaderos de Cuba, Costa Rica, Indonesia y Venezuela. Información de laboratorio mostró que el $27.4 \%$ de las vacas inseminadas no ll egó a concebi $r$ y los cel os subsiguientes no fueron observados, lo que ocasionó que su estado deno-preñez fuera identificado en el momento del diagnóstico degestación, usual mente real izado entre los 80-120 días del servicio. Adicionalmente se encontró que un $10.1 \%$ de animales perdieron el embrión entrelos 15 y 60 días del servicio. Los resul tados indicaron quecerca dela mitad del os servicios estuvieron asociados con deficiencias en el manejo reproductivo de los rebaños, ocasionando mermas importantes en la eficiencia de los Servicios de IA.

${ }^{1}$ Trabajo traducido por el autor y publicado original mente como: García, M., W. Goodger, T. Bennett, B.M.A.O. Perera. 2001. Use of a standardized protocol to identify factors affecting the efficiency of artificial insemination services for cattle through progesterone measurement in fourteen countries. En: Proceedings of theFinal Research Coordination Meeting of theFAO/OAEA Coordinated Research Programmeon «Use of RIA and Related Techniquesto I dentify Ways of Improving Artificial Insemination Programmes for Cattle Reared under Tropical and Sub-tropical Conditionss, IAEA, Viena, 2001

${ }^{2}$ Centro de Investigación IVITA,Universidad Nacional Mayor de San Marcos, Facultad de Veterinaria y Zootecnia, Universi dad Peruana Cayetano Heredia, Lima.

${ }^{3}$ Universidad de Wisconsin-Madison,Wisconsin, USA

${ }^{4}$ Sección de Producción y Salud Animal, Organismo Internacional deEnergía Atómica, Viena, Austria 
INTRODUCCIÓN

Establ os lecheros y al gunas ganaderías dedoblepropósito (lechey carne) utilizan en formarutinarialatécnica deinseminaciónartificial (IA) para el cruzamiento y el mejora miento genético desus animales. Sin embargo, se tiene un gran número de factores biológicos, gerenciales y socio-económicosque afectan la cal idad y la eficiencia de la técni$\mathrm{ca}$, que en al guna forma limita la participación de nuevos usuarios. Por otro lado, acciones encaminadas amejorar la eficienciade la IA tienen que basarse en el conocimiento adecuado delas condiciones prevalecientesen el medioy las limitaciones locales.

Larecolecciónsistemáticay detalladade información concemienteal sistemadeproducción, características de los predios ganaderos, comportamientoanimal, uso delatécnicadela IA, y dela expresión decelo puedepermitir la identificacióndelosfactoreslimitantesque $f e c$ tan la eficiencia del comportamiento reproductivo delos rebaños. Además deeso, la medicióndelosnivelesdeprogesteronaenmuestras de leche (o sangre en caso de vaquillas o vacas de carne) por el método del radioinmunoensayo (RIA) puedecontribuir con valiosa información para evaluar el estado reproductivo durante y después delal $\mathrm{A}$, y de terminar los factores que se encuentran afectando su eficiencia. EI RIA es además una he rramienta de importancia para monitorear las respuestas aintervencionestécnicas destinadas aaliviar las deficienciasencontradas enlosservicios delA (García1997; Williamsy Mdeod, 1992; Williams y Esslemont, 1993. Los resultados deestas intervenciones pueden quedar re flejadasen unamejora delaeficienciaenladetección decelo, unamejoraenel momentooportuno para realizar laIA, y un mayor porcentaje de concepción. Un beneficio adicional podría serlaidentificación deanimalescon problemas patológicos tales como situaciones de anestro después de los 60 días del parto.

El presentetrabajo muestra una síntesis de la metodología utilizada y los resulta- dos obteni dos bajo el Proyecto Coordinado delnvestigaciónFAO/OIEA intitulado “Uso del RIA y Técnicas Afines para Identificar Formas deM Mejorar los Programas del nseminación Artificial en Ganado Bovino Criado bajo Condiciones deTrópico y Subtrópico", el cual fue implementado en 14 países en desa rrollo en un periodo de 4 años. El objetivo de estetrabajo fuederecopilar información rele vante de cada uno de los proyectos nacionales afin deidentificar problemas comunes, y resal tar las ventajas y el potencial delametodología utilizada (entre ellas, la técnica del RIA, datos clínicos, formularios de entrada de datos y una base de datos) para la mejora dela eficiencia de los sistemas delA en países en desarrollo. Por otro lado, es importanteenfatizar que estetrabajo no tuvo por fina lidad comparar la eficiencia delos servicios delA ni resaltar diferencias entrepáses, sino quereflejan el estado delaIA en las localida des donde sehicieron los muestreos. Los autores son respetuosos de los derechos de los investigadores de cada pás sobre la informa cióncientífica obtenidaen el desarrollo deeste proyecto, y solicitan al lector a referirse a los trabajosindividualespublicadosene documento detrabajo para los detal les pertinentes ${ }^{3}$.

\section{MateRIALES Y MÉTODOS}

a) Selección y apoyo técnico a las instituciones participantes

Ladescripción completadel proyecto incluyendo el propósito, metas, metodología de trabajo y los requerimientos para participar fue publicada en el animal production and Health Newsl etter, J oint FAO/IAEA (1994) y distribuidos a nivel mundial a través de varios mecanismos incluyendo las oficinas de laFAO y del PNUD en los países en desarrollo. Serecibieron cerca de 30 propuestas, mayormentedeinstituciones deAsiay América Latina. Laselección delas instituciones participantes se hizo con base a capacidad técnica, víncul os apropiados con servicios delA, y disponi bilidad y expe- 
riencia en medición de progesterona por RIA. Lasinstitucionesseleccionadasy que les fueron asignadas contratos deinvestigaciónfueron de: Bangladesh(BGD), China (CPR), Indonesia (INS), Myanmar (MYA), Pakistán(PAK), Sri Lanka(SRL), y Vienam(VIE) en Asia $(n=7)$, y deArgentina (ARG), Chile (CHI), Costa Rica (COS), Cuba (CUB), Perú (PER), Uruguay (URU), y Venezuela(VEN) enAmé rica Latina $(n=7)$.

El Investigador Principal decadacontrato de investigación participó en 3 reuniones de coordinación. La primera se llevó a cabo en Noviembre de 1995 en Viena, Austria para estandarizar los pla nes detrabajo y coordinar actividades cla ve, así como un entrenamiento en el uso deAIDA (Artificial InseminationDatabase Application), paquetedecómputo desarroIlado específicamente para este proyecto (García, 1996). La segunda reunión se real izó en Melbourne, Australia enfebrero de1997 para revisar los resultados preliminares y definir las intervenciones técnicas. LatercerareuniónfueenUppsala, Suecia en Mayo de 1999 para presentar los resul tados finales y preparar los tra bajos para su publicación. El proyecto contó además con la participación deexpertos internacionales en los campos de la reproducción animal, inseminación artificial, evaluación de semen, y radioinmunoensayo, que proveyeron de apoyo técnico a los investigadores participantes durantelas reuniones y por contacto electrónico durantee desarrollo del proyecto.

Algunos delos autores deestedocumento visitaronen di versas oportunidades las localidades durante el desarrollo de los tra bajos. Copias delos archivos el ectrónicos de las bases de datos fueron reci bi das por $\mathrm{el}$ autor para su revisión y asesoramiento técnico delosinteresados. b) Recolección dela información

Las actividades de campo se desarroIlaron mayormentedurante1996 y 1997, aunque en al gunas locali dades se continuaron durante1998. El trabajo delaboratorio seconcluyó en Mayo de 1999.

Serecol ectó información concermiente a las fincas, inseminadores, semen utilizado, toros, vacas inseminadas, características de la expresión de celo observadas por los ganaderos y los inseminadores, y aspectos relacionadosa laIA. Se preparó cinco formularios para la toma de información (finca, inseminador, lote de semen, vaca, inseminación). Estos formularios, que fueron traducidos a los idiomas y dial ectoslocales, fueron diseñados ental forma que la información se tomaba marcando opcionesen listas, dejando una mínima parte para ser escrita. Todos Ios formularios se asemejaban a la disposición de los campos de la base de datos en la pantalla para facilitar su digitación. El listado de variables registradas, copias de los formularios, y explicación detallada de los mismos se encuentra en García 1996. Se esperaba queed trabajo abarcaraun mínimo de 500 vacas por país. Sin embargo, ARG, CUB, MYA y PAK no llegaron a cubrir esta meta Lasfincasfueronseleccionadas con el criterio de ser representativas del sistema deproducción del medio. Fincas élite o aquellas con niveles productivos o reproductivos muy superioresal promedio nofueron consideradas. Losinseminadores no fueron informados de los objetivos reales deesteestudio afin deevitar sesgos en los resultados debidos a cambios en sus actitudes y rendimientos.

c) M uestreo de leche (y sangre) y análisis delaboratorio

Se recolectaron tres muestras, iniciándose el proceso en el primer 
serviciopostparto(odesuvidareproductiva encaso deservaquilla). Laprimerasetomó en el día del servicio (día 0), la segunda entre los días 10-12 postservicio, y la terceraentrelos días 22-24 postservicio. La tercera muestra se colectaba únicamentesi $\mathrm{e}$ animal no era observado en celo dentro del tiempo esperado. Este esquema de muestreo fue repetido en serviciossubsecuentes enal gunos páses.

Una muestra típica de leche consistió en 5-20 ml de leche recolectada de cual quier cuarto mamario sano. Laleche secolectaba en viales devidrio o plástico que contenían azida de sodio como preservante, se guardaban en refrigeración $\left(+4^{\circ} \mathrm{C}\right)$ por 3-7 días en quese separaba la porción grasa por centrifugación (2000 g/15 min). Las muestras desangre (5-10 m) fueron usualmente obtenidas por punción de la vena yugular en vacutai ners heparinizados, y el plasmase obtuvo por centrifugación dentro de las 4 horas dela colección. Las muestras de leche desgrasaday de plasma seguarda ron a-20C hasta su análisis.

La concentración de progesterona en las muestras delecheo plasmasemidieron utilizando el kit de RIA FAO/IAEA (Plaizier, 1993), el cual estábasado en una técnicadeRIA defase sólidaqueemplea 125I-progesterona como marcador.

d) Almacenamiento de la información y análisis estadístico

Una base de datos ad hoc con repal do en MicrosoftAccess 2.0 y consuspropios archivos deejecuciónfuedesarrollada para el ingreso de la información de campo y de laboratorio. Este software intitulado "AIDA" (Artificial InseminationDatabase Application" esta compuesto de hojas de entradadedatosparafincas, inseminadores, lotes de semen, vacas inseminadas, servicios, y valores de progesterona. La aplicación contiene facilidades para la verificación dela información y un gran número deinformes preestablecidos con análisis estadísticos simples para el cál culo del comportamiento reproductivo y la interpretación de los valores de progesterona. AIDA también contiene rutinas para la exportación de una amplia variedad de datasets quepermiten agrupar datos crudos y cal culados bajo un formato deExce 3.0.

Los análisis estadísticos utilizados en este estudio están descritos en GAIDA (Guide for AIDA Data Analysis). (Goodger et al., 1998)

\section{Resultados y Discusión}

Características de las fincas y los servicios delA

a) Fincas

Las instituciones participantes de los países asiáticos, con excepción de CPR, trabajaron con predios pequeños (1-2 vacas) aunque se llegó incluir algunos predios de 10-20 vacas. La información deINS y SRL incluyó dostipos defincas (predios pequeños y ganaderías grandes), de modo que el análisis de realizó por separado. El tipo predominantedeganado fueel detipo lechero, seguido deganado de doble propósito (Cuadro 1), y en el caso de INS fue ganado de carne. El ordeño fue hecho mayoritariamente a mano 1-2 veces al día, utilizando al temero para el apoyo de la "bajada de la leche". Registros a nivel de finca eran inexistentes o limitados a tarjetas individuales.

Losestudiosen paíseslatinoamericanos se hicieron en fincas grandes con poblaciones quevariaron entre 20 a 1,500 vacas, principal mentedetipo Holstein. El ganado se mantuvo en corrales $(\mathrm{CHI}$, COS, CUB, PER) o en potreros (ARG, URU, VEN). Se hizo ordeño mecánico dos veces al día sin la presencia del temero (Cuadro 1). El uso de registros 
Cuadro 1. Características predominantes de las fincas utilizadas en los 14 países participantes del proyecto

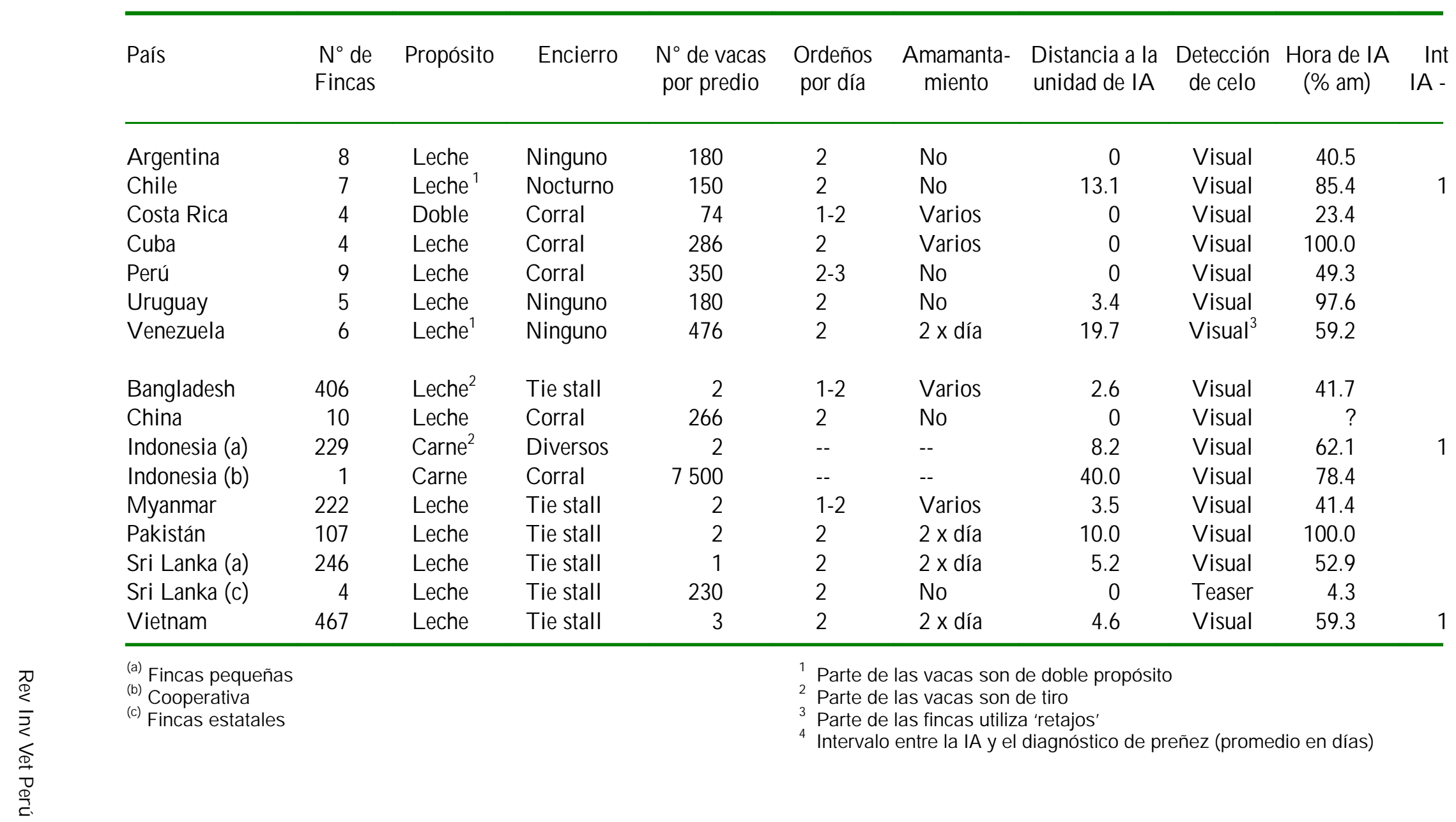


electrónicos y registros en fichas individual es fueuna práctica común.

b) Unidades deInseminaciónArtificial

Los ganaderos utilizaron diversos mecanismos paracontactar al as unidades deinseminación. Enel caso defincasen América Latina, losinseminadorestenían que cubrir grandes distancias con excepción de aquellas que tenían sus propios técnicos (CHI, VEN), en tanto que en Asia, las fincas se encontraban entre 1 a $7 \mathrm{~km}$ de las unidades de IA (Cuadro 1). Los inseminadores se desplazaban en bicicletas, motos o automóviles.

El tiempo que transcurrió entre el momento enqueserealizólainseminación con relaciónal momento enquesedetectó el celo no tuvo un patrón definido. Todos losserviciosen PAK y CUB y lamayoría delosserviciosenCHI yURU serealizaron en las mañanas (Cuadro 1), mientras que en otras localidades se observó una distribución similar entre los servicios de la mañana y la tarde. El intervalo entrela detección del celo y la inseminación fue registrado en 7 países, encontrándose interval os demesiado amplios (BGD 17.6 h, SRL 20.9hy VIE 22.4h). El diagnóstico depreñez por pal pación rectal sehizoentre los 80 a 120 días del servicio sin retomo (Cuadro 1).

\section{c) Técnicosinseminadores}

El número deinseminadores por estudio varió entre 2 a 19 (promedio: 8.3 y mediana: 7). Losinseminadores depaíses asiáticosfueron mayormenteempleados de centrales de IA dependientes del estado, en tanto que en América Latina eran empleados de las mismas fincas o de cooperativas privadas.

Se encontró una gran variabilidad en el nivel educativo así como en el número promedio de inseminaciones mensuales por inseminador (Cuadro 2).

d) Semen y toros

El semen utilizado fue producido local mentecon excepción dePER y COS que utilizó semen importado. Semen congel ado como pel let fue utilizado en forma parcial en CPR y VIE y exclusiva en CUB, y en la forma de pajillas en los demás países. Semen refrigerado fue utilizado en forma limitada en BGD y SRL. Una observación interesante fue encontrar quelos servicios del A en Asia utilizan pajillas de $0.25 \mathrm{ml}$ mientras que en América Latina se utilizan pajillas de $0.5 \mathrm{ml}$ (Cuadro 2). Ladescongelación de semen se hizo principalmente en agua tibia.

El número detoros ayyosemenseutilizó en los estudios varió entre 7 a 68 por pás (promedio 29.9, mediana 25, Cuadro 2). Lacalidad del semen, en los casos quefue evaluado, estuvo usual mentedentro delos estándares esperados, con excepción de PAK, donde la motilidad y viabilidad de los espermatozoides fue bastante baja debido al manejo y almacenamiento inapropiado en las unidades delA.

e) Vacasinseminadas

La edad al primer servicio postparto en lapoblación bajo estudio fuede $5.6 \pm 2.3$ años (Cuadro 3, promedio \pm d.e., $\mathrm{n}=$ $5,476)$ con un número de partos de $2.8 \pm$ $1.7(n=5,953)$, sin que se haya encontrado diferencias estadísticas significativas entre países. Esta homogeneidad entreedad al primer parto y número de parto facilitó las eval uaciones de otras variables.

La necesidad de prestar asistencia veterinariaen el partofuenotoriaenARG (16.7\%), CHI (30.2\%) y URU (11.5\%). Colocar vacas próximas al parto en corrales deobservación continuadalas 24 
Cuadro 2. Características predominantes del semen utilizado y de los inseminadores en los 14 países participantes del proyecto

\begin{tabular}{|c|c|c|c|c|c|c|c|c|c|}
\hline \multirow[b]{2}{*}{ País } & \multicolumn{5}{|c|}{ Semen } & \multicolumn{4}{|c|}{ Técnico inseminador } \\
\hline & $\begin{array}{l}\mathrm{N}^{\circ} \mathrm{de} \\
\text { toros }\end{array}$ & $\begin{array}{l}\mathrm{N}^{\circ} \text { de } \\
\text { lotes }\end{array}$ & Origen & $\begin{array}{l}\text { Tipo de semen } \\
\text { (volumen, } \mathrm{ll} \text { ) }\end{array}$ & $\mathrm{N}^{\circ}$ & $\begin{array}{l}\text { Edad } \\
\text { (años) }\end{array}$ & Nivel deeducación & $\begin{array}{l}\text { IA por } \\
\text { mes }^{1}\end{array}$ & Emplead \\
\hline Argentina & 30 & 32 & Local $^{2}$ & Pajilla (0.25) & 10 & 40.0 & Secundaria / Primaria & 23 & Finca privada / e \\
\hline Chile & 52 & 65 & Local $^{2}$ & Pajilla $(0.5 / 0.25)$ & 6 & 48.5 & Técnica / Primaria & 195 & Independiente / d \\
\hline Costa Rica & 11 & 15 & Importado & Pajilla-? & 4 & 28.2 & Primaria & $?$ & Finca privada \\
\hline Cuba & 19 & 20 & Local & Pellets (0.5) & 7 & 33.3 & Técnica & 150 & Finca estatal \\
\hline Perú & 68 & 68 & Importado & Pajilla (0.5) & 19 & $?^{5}$ & Diversa & 20 & Finca privada \\
\hline Uruguay & 24 & 24 & Local $^{2}$ & Pajilla (0.5) & 3 & 39.6 & Primaria / Superior & 135 & Finca privada/ e \\
\hline Venezuela & 51 & 60 & Local & Pajilla (0.5) & 10 & 36.3 & Secundaria/Primaria & 90 & Finca privada/co \\
\hline Bangladesh & 26 & 145 & Local $^{2}$ & Pajilla/Refrigerado 3 & 7 & 39.9 & Técnica & 165 & Centro estatal de \\
\hline China & 67 & 67 & Local & Pajilla/Pellets (0.25) & 8 & 34.2 & Secundaria/Profesional & $?$ & $?$ \\
\hline Indonesia (a) & 6 & 14 & Local & Pajilla (0.25) & 14 & 29.1 & Secundaria / Primaria & $?$ & Centro estatal de \\
\hline Indonesia (b) & 2 & 5 & Local & Pajilla (0.25) & 2 & $?$ & Secundaria & $?$ & Centro estatal de \\
\hline Myanmar & 11 & 45 & Local & Pajilla (0.25) & 5 & 48.4 & Técnica & 65 & Centro estatal de \\
\hline Pakistán & 7 & 7 & Local & Pajilla (0.5) & 2 & 28.8 & Técnica & 35 & Centro estatal de \\
\hline Sri Lanka (a) & 24 & 68 & Local $^{2}$ & Pajilla/Refrigerado 4 & 8 & 42.9 & Secundaria & 54 & Centro estatal de \\
\hline Sri Lanka (c) & 7 & 7 & Importado & Pajilla (0.25) & 7 & 34.2 & Secundaria & 43 & Finca estatal \\
\hline Vietnam & 13 & 13 & Local $^{2}$ & Pellets/Pajilla (0.5) & 4 & 35.5 & Profesional / Técnica & 200 & Centro estatal de \\
\hline
\end{tabular}

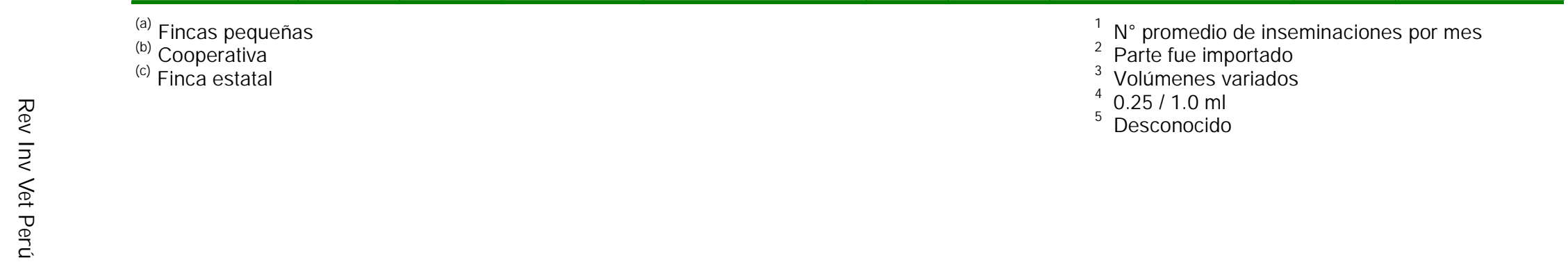


horas, y realizar laintervención veterinaria en casos queel parto demoremés de 1 a 2 horasesunaprádicacomúnenestos países. Unaaltaincidenciaderetencióndeplacenta fue observada en VIE (9.9\%).

En el estudio realizado en CUB se observó una pobre condición corporal y baja producción de leche (1.9 y $4.8 \mathrm{~kg}$, respectivamente) en vacas Holstein de crianza intensiva debido a la falta de concentrados y escasez de forrajes en el país. El peso corporal enel díadel servicio varió grandemente entre países debido principal mente a la variedad de razas y regímenes nutricionales. El mayor peso corporal se observó en PER (591kg), CHI (546 kg) y URU (536 kg), y los más bajos en BGD $(211 \mathrm{~kg})$, el cual reflejaba el tamaño pequeño delos animaleslocales (Cuadro 3).

\section{f) Expresión decelo elA}

La ocurrencia de descarga mucosa en la zona vulvar fue el signo de celo más utilizado como signo decelo en los países asiáticos consistemas demanejotipo ‘tie stall'. Signos como que la vaca monta a otra o que se deja montar fueron importantes en fincas que tenían varias vacas (Cuadro3). Por otrolado, en fincas latinoamericanas, la vaca que se deja montar fue el signo representativo por excelencia de la vaca en celo, de modo que otro tipo de sintomatología fue raramente aceptada. Sincronización de cel o fue utilizadaen al gunos animales de INS y en la mayor parte de los animales deCUB.

Los inseminadores reportaron mayoritariamente que la vulva se encontrabaedematosaen el momento del servicio. El nivel dedificultadenel pasaje dela pi peta (fácil, difícil, imposible) fue reportada como difícil en $>10 \%$ de los servicios en BGD, CHI, IND (finca estatal) y VIE (Cuadro 3).

\section{Comportamiento R eproductivo}

El Cuadro 4 muestrael comportamiento reproductivo de las vacas inseminadas en los 14 países. La diferencia en el número de observacionesentree intervalo al primer servicio $(n=7,991)$ y el porcentaje de concepciónal primer servicio $(n=8,196)$ fuedebido por un lado a la ausencia de las fechas de parto en los registros y por otro lado, a la dificultad dehacer los di agnósticos de preñez en al gunos países. CPR tuvo la mayor cantidad de observaciones (2,018 vacas) y PAK tuvo la menor cantidad (110 vacas).

El promedioglobal del intervaloentreed parto al primer servicio fue de $121.5 \pm 82.1$ días (mediana $=95$ días) con grandes diferenciasentrepáses. Losinterval os més cortosocur mieron en PER, CHI y ARG, debido probable menteala mejor cal idad genéticadelosanimales, mayor nivel de alimentación y manejo, y mejorescondiciones medio-ambientales parala crianza de ganado Holstein. El largo intervalo observado en CUB puedeser atribuido al deficienteplano nutricional queseencontraban los animales debido a los problemas económicos queseencontrabanel paísdurantelaejecución del estudio. Este intervalo fue más largo en la mayoría delos páses asiáticos, especialmente cuando setrabajóconganado nativo en BGD y PAK (cercade200 días) y conganado decame en INS (cerca de270 días).

El promedio global de interval o entre el parto alaconcepciónfueúnicamentede 16 días másqueed interval o entreel parto al primer servicio. Esta diferencia entre los promedios tiene que ser tomada con cautela ya queel promedio del intervalo ala concepción esta dado por menos del $50 \%$ dela población inseminada. Muchas vacas no llegaron aquedar gestantes y fueron descartadas delas fincas, y otras quedaron gestantes pero en servicios posteriores y queno fueron partedel presenteestudio.

El promedio del porcentajedeconcepción al primer servicio (CPS) fue de $40.9 \%$. 


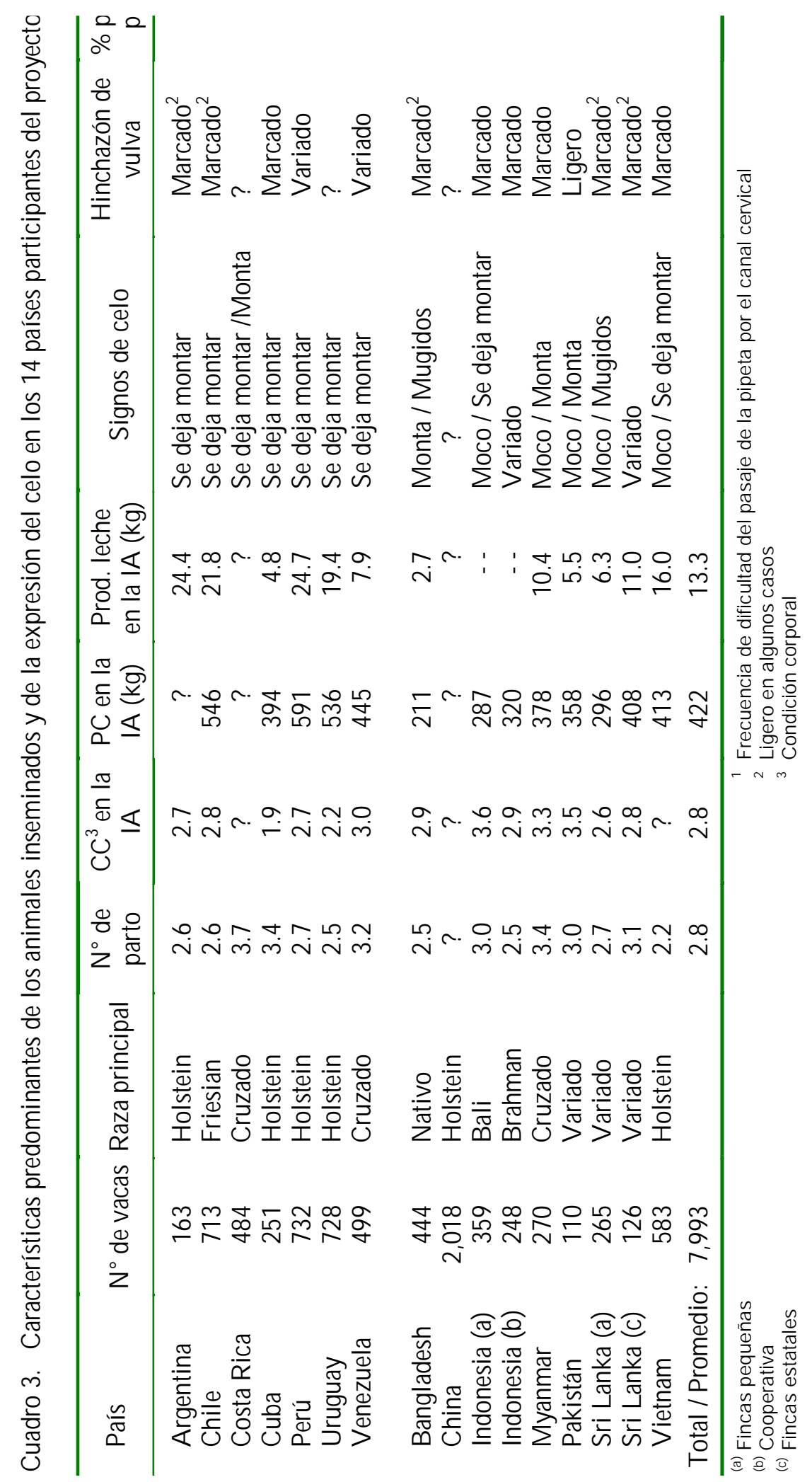


Cuadro 4. Intervalos (días) entreel parto al primer servicio y a la concepción, y porcentaje de concepción al primer servicio en lo participantes del proyecto

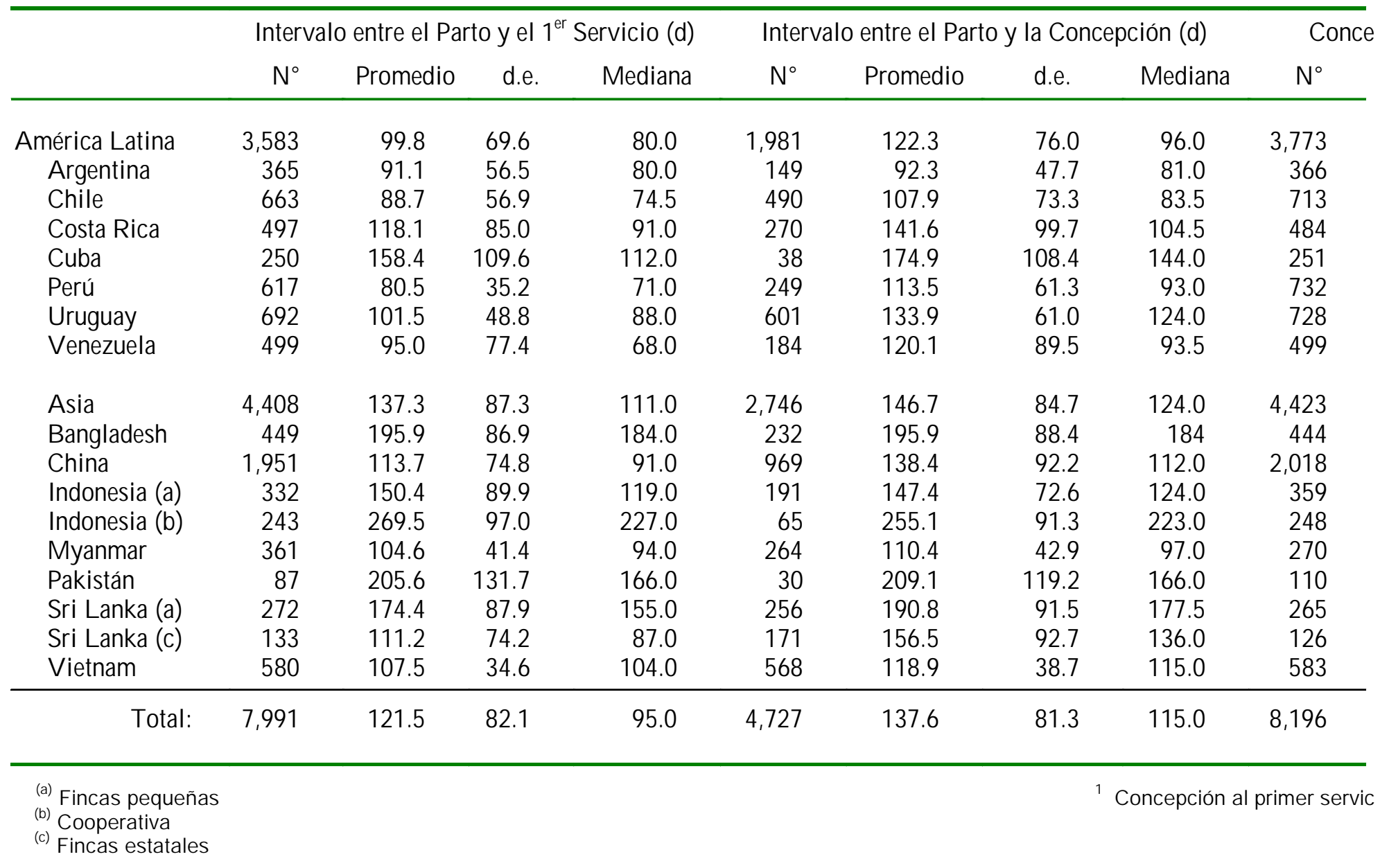


El porcentajedeconcepción detodos los servicios no fue calculado para el propósito de esteanálisis debi do a queno sehizo el seguimiento reproductivo a una elevada porción deanimalesqueretornaron en celo. El mayor CPS ocurrió en VIE (62.1\%), CHI (61.9\%) y MYA (58.9\%) mientras que los obteni dos en CUB, INS, PAK, y PER fueron los más bajos. Las razones atribuibles al bajo comportamiento reproductivo deCUB fueron previamente descritos y solo habría que añadir que se hizo sincronización de celo en vacas con pobre condición corporal. En PAK fue debidoal deficientemanejo y al macenamiento de las pajillas de semen en las unidades de IA, mientras que los animales del PER estur vieron bajo los efectos del estrés decal or ocasionados por el fenómeno de “El Niño" que causó problemas demuerteembrionariatemprana y anestro subsecuente (Armstrong, 1994). En el caso deINS (en ambos tipos de fincas) se pudo demostrar, como severá más adelante, una alta proporción de animales inseminados durantela faseluteal.

Las diferencias en CPS debidas a ta maño de finca, condición corporal en la IA, facilidad del pasaje de la pi peta, años deexperiencia del inseminador, grado de edema vulvar en el momento del servicio, y el origen del semen pudieron ser evaluadas en países que di sponían de un número balanceado entregrupos. Las fincas de VIE y MYA con 12 vacas o con $5-20$ vacas no difirieron en CPS, mientras que fincas grandes (>300 va cas) en COS y VEN en COS y VEN mostraron tasas de concepción inferiores (14.8 y $22.2 \%$, respectivamente) en comparacióncon fincas pequeñas (20-300 vacas, 44.4 y 53.3\%, respectivamente) ( $P \varangle 0.05$ ). Las diferencias en $\mathrm{CHI}$ entrefincas de tamaño medio (47\%) y fincas grandes (56.0\%) no fueron significativas. Lacondición corporal enel día dela IA fue un factor importante cuando se combi nó la información de los estudios realizados en BGD, CHI, SRL y VEN (Figura 1, n $=1,494$ ). Las vacas con un val or de 1.0-2.4 (en una escal a de 1-5) tuvieron un CPS inferior $(33.1 \%, \mathrm{P}<0.05)$ quevacas con una CC de $2.5-3.5$ (40.0\%) y >3.5 (40.4\%).

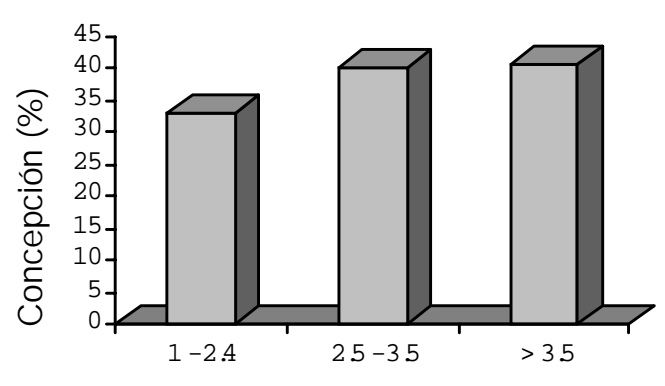

Condición corporal en el día de la IA

Figura 1. Efecto de la condición corporal (1-5) en el porcentaje de concepción al primer servicio en ganado lechero y de doble propósito de Bangladesh, Chile, Sri Lanka y Venezuela

Información combinadadeBGD, SRL, VIE, ARG, CHI, COS y CUB $(n=2,330)$ mostró diferencias en CPS ( $P \varangle 0.05$ ) entre servicios llevados a cabo por inseminadores con $<7$ años deexperiencia ( $24.9 \%$ ) y aque Ilos con 7.1-12.0 años (44.2\%) y > 12 años (43.5\%). El grado deedemavulvar enel momento delalA (ninguno, ligero, marcado) no mostró una relación de importancia con el CPS ( $n=1,519, P>0.05$ ), en tanto que $\mathrm{d}$ semen local vs semen importado tuvieron un comportamiento similar enSRL y URU, pero más bajo en BGD (38.9 vs. 57.5\%, respectivamente, $\mathrm{P} \varangle 0.05$ ).

El intervalo entreed primer y segundo servicio en 1,959 observaciones fue de 44.6 \pm 44.4 días. Esto es el equival ente a la ocurrencia de un cel o no observado entrelos dos servicios. Serios problemas de detección de cel o fueron observados en COS y CPR donde este interval o fue de $>57$ días.

Problemas identificados en los Servicios de IA

Básicamente, la muestra tomada en el día del servicio fue usada para verificar si el animal fueinseminado durantela faseluted (es decir, enel momento inapropiado, altaconcentración de progesterona); el juego de dos muestras (muestra en el día del servicio y muestra a mitad deciclo) fueusado para ve rificar si el animal estaba ciclando y si había ovulado; y el juego de tres muestras más el 
diagnóstico depreñez (latercera muestracolectadaenel día 22-24 del servicio) fueutilizado para verificar si el animal había concebido, perdido el embrión, osi sehabíainseminado estando gestante o en fase de acidia La pre sentación de niveles más frecuentes de progesterona y su interpretación se indican en el Cuadro 5.

Dos deficiencias deimportanciafueron identificadasen los Servicios delA através del análisis de progesterona en muestras colectadas en más de 8,500 inseminaciones (Cuadro 6). Inseminacionesen momentos inapropiados serealizaronenel $17.3 \%$ deloscasos, denotandoserioserroresen detección deceloanivel de finca. Estos servicios se hicieron en vacas que no estaban ciclando (10.4\%) y en vacas con presencia de cuerpo lúteo (CL) activo (6.9\%), estando preñadas la mayoría de estas vacas. Inseminaciones en vacas que no estaban ciclando fueron más frecuentes en CUB, INS (en ambostipos defincas), COS y VEN (Cua droVII). Serviciosrealizadosen vacas con CL activo sered izaron mayormenteen fincasestatales deINS (17.8\%), fincas pequeñas deSRL (13.2\%) y enURU (11.8\%).

Lasegundamayor deficienciaestuvore lacionada conla detección decelo postservicio y el manejo del rebaño, el cual afectóal 37.5\% delasinseminaciones(Cuadro6). En estecaso, e $10.1 \%$ de los animales inseminados sufrieron muerte embrionaria (entre 16-60 días postservicio) pero no fueron identificadas hastaqueserealizóel diagnóstico depreñez, usualmente80-120 días después del servicio. Otro grupo querepresentó el $27.4 \%$ de los animalesinseminados fallóen concebir peroloscelossubsiguientes no fueron observadosy permanecieron es eseestado hastaqueserealizó el diagnóstico de preñez. Fincas de CPR y URU tuvieron la mayor frecuencia de muertes embrionarias (17\%), mientras que fincas de CUB, PER y INS tuvieron las mayores frecuencias de vacas con cel os no observados después de inseminaciones no exitosas $(68.9,45.3$, y cerca de $40 \%$, respectivamente).
Funcionalidad y limitaciones de la es trategia de tres muestras

El juego de 3 muestras deleche (o sangre) recolectadas en los días 0, 10-12, y 2224 de la inseminación artificial, junto con e resultado del diagnóstico de preñez por recto-pal pación demostró ser una estrategia de mucha utili idad para la eval uación de la eficienciadel manejo reproductivo anivel defincay delos resultados del Servicio delA. Sin embargo, es importante señalar que el sistemadetrabajo requiere degran número deanimales para quela interpretación de los resultados pueda ser consistente. El número deanimales dependerá, entreotrosfactores, del tipo definca, dela variabilidad genética delapoblación, del número devariables a ser considerado en el análisis, y la eficiencia en la re colección demuestras.

La toma de la primera muestra no re quiere de mayor trabajo y puede ser hecha por el propio inseminador. Las otras dos muestras requieren queal guien visitelas fincas, o seatomada por el propio ganadero (fincas pequeñas) o empl eados delasfincas (fincas grandes) en el día exacto y en los anima les debidos. De ser tomada por personal de las fincas, se requiere tener un mecanismo apropiado para remitir las muestras al laboratorio. Una segunda limitación estriba en queun $18.4 \%$ de los juegos de 3 muestras de esteestudio $(n=6,483)$ tuvieron por lo menos una muestra con valores intermedios de progesterona ( $>a<3 \mathrm{nmoL}$ ), y por lo tanto el set quedó inval idado para real izar una interpretación adecuada. Buenas prácticas de laboratorio en el uso del RIA pueden reducir estos inconvenientes aunque otros factores como el mal manejo de las muestras pueden contribuir a magnificar este problema.

\section{Conclusiones}

El uso dela metodología estandarizada entre países y la estrategia de combinar información decampo con val ores deprogesterona, aunado a los formularios para el manejo de la 
Cuadro 5. Interpretación de datos de RIA de Progesterona solos o en combinación con información clínica para la eval uación de los servicios de IA (Alto : $\geq 3 \mathrm{nmol} / \mathrm{l}$; Baj o: $\leq 1 \mathrm{nmol} / \mathrm{l}$ ) usando muestras de leche (o plasma) recolectadas a diferentes interval os Post IA

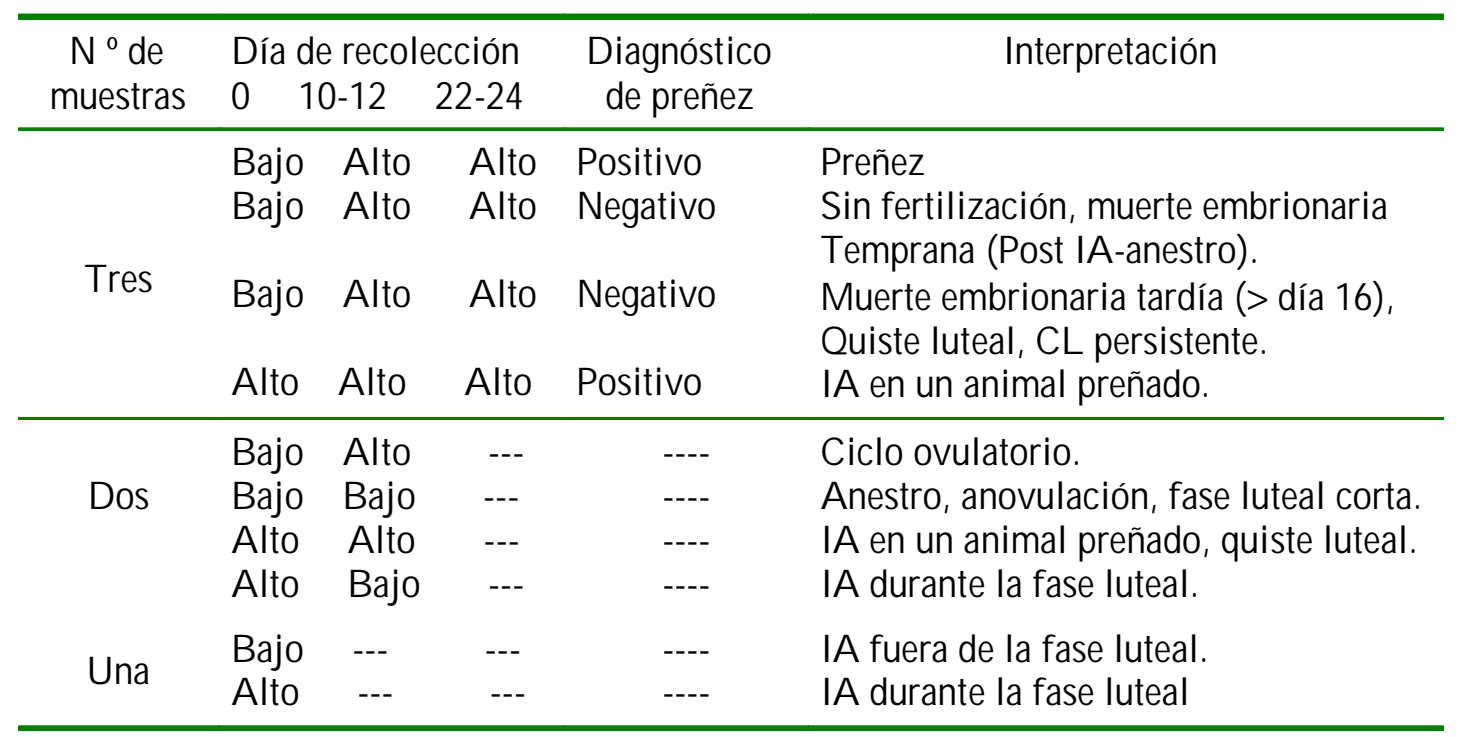

Cuadro 6. Factores detrimentales del servicio de IA que han si do identificados a través de la estrategia de 3 muestras y el diagnóstico de preñez

\begin{tabular}{|c|c|c|}
\hline Problema principal & $\begin{array}{c}\text { Deficiencia espećfica en } \\
\text { el servicio delA }\end{array}$ & $\begin{array}{c}\text { Población afectada } \\
\text { ( \% ) }\end{array}$ \\
\hline \multirow{2}{*}{$\begin{array}{l}\text { - IA inapropiada } \\
\text { probablemente debido a } \\
\text { detección incorrecta de celo }\end{array}$} & & 17.3 \\
\hline & $\begin{array}{l}\text { - IA en vacas con cuerpo lúteo activo } \\
\text { - IA en vacas queno están ciclando }\end{array}$ & $\begin{array}{r}6.9 \\
10.4\end{array}$ \\
\hline \multirow{3}{*}{$\begin{array}{l}\text { - Detección de celo y manejo } \\
\text { derebaño deficientes }\end{array}$} & & 37.5 \\
\hline & $\begin{array}{l}\text { - Vacas quefallan en concebir, el celo } \\
\text { subsiguiente no es detectado y se } \\
\text { encuentran vacías al diagnóstico de } \\
\text { preñez. }\end{array}$ & 27.4 \\
\hline & $\begin{array}{l}\text { - Vacas que conciben pero pierden e } \\
\text { embrión y se les encuentra vacías en } \\
\text { e diagnóstico de preñez. }\end{array}$ & 10.1 \\
\hline
\end{tabular}


información decampo y la basededatos, permitió la generación de información única en su género sobre el comportamiento reproductivo del bovino y los sistemas delA en paísesen desarrollo. Esto resul tó en un adecuado víncul o entre instituciones de investigación y centrales delA, y permitiólaidentificación delos principal es factores quelimitan la eficiencia de la IA en las local idades estudiadas en los 14 países asiáticos y de América Latina que participaron en el estur dio.

El estudio mostró que cerca de la mitad delos servicios estuvieron relacionadosa una deficiencia, mayormenteasociada aerror humano, y que afectaron severamented comportamiento reproductivo de los establecimientos pecuarios y por lo tanto, la cal idad del servicio de IA. Esfuerzos futuros deben estar abocados en al iviar la intensi dad delos problemasencontrados, y los cual es seincluyen los siguientes: educar al os ganaderosen detección decelo, y en prácticas demanejoy nutrición animal; mejorar el conocimiento y habilidades delos inseminadores enla práctica dela IA; ampliar la investigación en mortalidad embrionaria, amamantamiento, métodos deinducción osincronización decelo; me jorar a manejo, al macenamiento y control de calidad del semen; y mejorar el sistema de registro, evaluación y seguimiento de las inseminaciones.

Estudiossimilares serían degran utilidad en otros países en desarrollo para mejorar la eficiencia de sus servicios deIA.

Agradecimientos

Los autores expresan su sincero agra decimiento a los siguientes investigadores e instituciones por su importantecontribución al éxito de este programa de investigación: María-Elena Mongiardino (INTA-Castelar, Argentina), Mohammed Shamsuddin (Bangladesh Agricultural University, Bangladesh), Nestor Sepúl veda(Universidad
delaFrontera, Chile), Cai Zhenghua(Chinese Academy of Agricultural Sciences, China), Sandra Estrada (Universidad Nacional, CostaRica), Rodolfo Pedroso (CIMA, Cuba), A. Latief Toleng (Hasanuddin University, Indonesia), U Than HIa (Ministry of Livestock Breeding and Fisheries, Myanmar), Mohamed Anzar (National Agricultural Research Council, Pakistán), Luisa Echevarnía(Universidad Nacional Mayor de San Marcos, Perú), Harischandra A beygunawardena(University of Peradeniya, Sri Lanka), Danie Cavestany (INIA, Uruguay), Eleazar Soto-Bell loso (Universidad del Zulia, Venezuela), Chung Anh Dzung (Institute of Agricultural Sciences of South Vietnam, Vietnam).

Los autores agradecen además a los Drs. Carlos Galina (UNAM, México), Le Fitzpatrick (J ames Cook University, A ustra lia), David Galloway (University of Melbourne, Australia) y Mats Forsberg (SwedishUniversity of Agricultural Sciences, Suecia) por su asesoría y apoyo técnico duranteel desarrollo del programa.

\section{LITERATURA CITADA}

1. García, M., Uso del radioinmunoensayo de progesterona para mejorar la producción animal en sistemas de doble propósito. En: Conceptosy Metodologías de Investigación en Fincas con Sistemas deProducciónAnimal deDoblePropósito. Cali, Colombia. Centro Internacional de Agricultura Tropical (CIAT), Consorcio Tropileche (1997) 26-44.

2. Williams, M.E.; B.J. McLeod. 1992. Strategic milk progesteronetesting for the detection of silentestrus and anovulation in dairy cows. J. Agric. Sci., Cambridge 118: 237-244.

3. Williams, M.E.; R.J . Esslemont 1993. A decision support system using milk progesterone test to improve fertility in commercial dairy herds. Vet Rec. 5: 503506. 
4. Animal Production and Health Newsletter, J oint FAO/I AEA. Division of Nuclear Techniques in Food and Agriculture, International Atomic Energy Agency. 1994, N²0, Vienna, 27p.

5. García, M. 1996. User manual for AIDA (Artificial Insemination Database Application) 36p.

6. Plaizier, J .C.B. 1993. Validation of the FAO/IAEA RIA kit for measurement of progesterone in skin milk and blood plasma. In: Improving the Productivity of Indigenous African Livestock. (Ed. IAEA), Vienna: IAEA-TECDOC-708pp. 151-156.

7. Goodger, W.J .; M. García; M. Clayton; T. Bennett; C.B., Thomas, C., Eisele, Guide For AIDA Data Analysis. IAEA, Vienna, (1998) p.37.

8. Armstrong, D.V. 1994. Heat stress interaction with shade and cooling. J . Dairy Sci. 77: 2044-2050. 